\title{
A retrievable implant for the long-term encapsulation and survival of therapeutic xenogeneic cells
}

\author{
Suman Bose $\mathbb{D}^{1}$, Lisa R. Volpatti1,2,9, Devina Thiono, 1,3,9, Volkan Yesilyurt ${ }^{1,3}$, Collin McGladrigan ${ }^{1,3}$, \\ Yaoyu Tang1,3, Amanda Facklam ${ }^{11}$, Amy Wang ${ }^{2}$, Siddharth Jhunjhunwala ${ }^{1,7}$, \\ Omid Veiseh ${ }^{1,8}$, Jennifer Hollister-Lock ${ }^{4}$, Chandrabali Bhattacharya', Gordon C. Weir ${ }^{4}$, \\ Dale L. Greiner ${ }^{5}$, Robert Langer $\mathbb{B}^{1,2,3,6}$ and Daniel G. Anderson $\mathbb{1}^{1,2,3,6 \bowtie}$
}

'David H Koch Institute for Integrative Cancer Research, Massachusetts Institute of Technology, Cambridge, MA, USA. ${ }^{2}$ Department of Chemical Engineering, Massachusetts Institute of Technology, Cambridge, MA, USA. 'Department of Anesthesiology, Boston Children's Hospital, Boston, MA, USA. ${ }^{4}$ Section on Islet Cell and Regenerative Biology, Research Division, Joslin Diabetes Center, Boston, MA, USA. ${ }^{5}$ Program in Molecular Medicine, University of Massachusetts Medical School, Worcester, MA, USA. ${ }^{6}$ Institute of Medical Engineering and Science, Massachusetts Institute of Technology, Cambridge, MA, USA. 'Present address: Centre for BioSystems Science and Engineering, Indian Institute of Science, Bengaluru, India. ${ }^{8}$ Present address: Department of Bioengineering, Rice University, Houston, TX, USA. ${ }^{9}$ These authors contributed equally: Lisa R. Volpatti, Devina Thiono. ${ }_{\text {e-mail: dgander@mit.edu }}$ 


\section{Table of contents}

\section{A Supplementary tables}

Atomic composition of device surface measured by XPS 2

B Supplementary figures

Fig S1. Design of the macrodevice 3

Fig S2. EPO secretion rate of free and encapsulated HEKepo cells 4

Fig S3. Proliferation and viability of cells inside the device $\quad 5$

Fig S4. Escape of encapsulated cells from macrodevices 6

Fig S5. Representative z-stack images of $0.6 \mu \mathrm{m}$ and $0.4 \mu \mathrm{m}$ devices $\quad 7$

Fig S6. Estimation of pore-size distribution $\quad 8$

Fig S7. XPS spectrum analysis of device surface after ATRP modification 9

Fig S8. Effect of surface coating on hydrophilicity of the device 10

Fig S9. Surface analysis of the device using SEM $\quad 11$

Fig S10: Measuring permeability of membranes $\quad 12$

Fig S11: Confocal raman mapping of devices after retrieval 13

Fig S12: Detection of graft specific antibody in serum of C57BL/6 mice 14

Fig S13: EPO secretion rate of retrieved devices $\quad 14$

Fig S14: Dox inducible EPO secretion rate of engineered cells $\quad 15$

Fig S15: Hematocrit of animals receiving inducible HEKepo cells $\quad 15$

Fig S16: Individual BG plots of Figure $6 . \quad 16$

Fig S17. IVGTT and insulin levels on Day $7 \quad 17$

Fig S18: Retrieval of transplanted devices carrying islets from animals $\quad 18$

Fig S19: Histological images of the retrieved devices carrying rat islets 19

C Estimation of membrane pore-size distribution 20 
Supplementary Table 1. Atomic composition of device surface measured using XPS (mean \pm s.e.m, $n=3)$.

\begin{tabular}{c|c|c|c|c|c|c}
\hline \hline Material & $\mathrm{C} \%$ & $\mathrm{O} \%$ & $\mathrm{Si} \%$ & $\mathrm{~N} \%$ & $\mathrm{~S} \%$ & $\mathrm{P} \%$ \\
\hline Native PDMS & $44.4 \pm 1.76$ & $28.3 \pm 1.8$ & $27.2 \pm 0.13$ & - & - & - \\
CBMA & $48.5 \pm 0.36$ & $26.19 \pm 2.4$ & $19.55 \pm 1.00$ & $5.75 \pm 2.88$ & - & - \\
SBMA & $56.73 \pm 2.01$ & $27.78 \pm 0.57$ & $9.24 \pm 2.01$ & $3.26 \pm 0.63$ & $3.14 \pm 0.35$ & - \\
MPC & $53.02 \pm 2.67$ & $30.57 \pm 1.68$ & $8.86 \pm 2.53$ & $3.57 \pm 0.56$ & - & $3.82 \pm 0.52$ \\
THPT & $60.6 \pm 0.31$ & $19.8 \pm 0.73$ & $9.6 \pm 1.25$ & $9.9 \pm 1.12$ & - & - \\
\hline
\end{tabular}




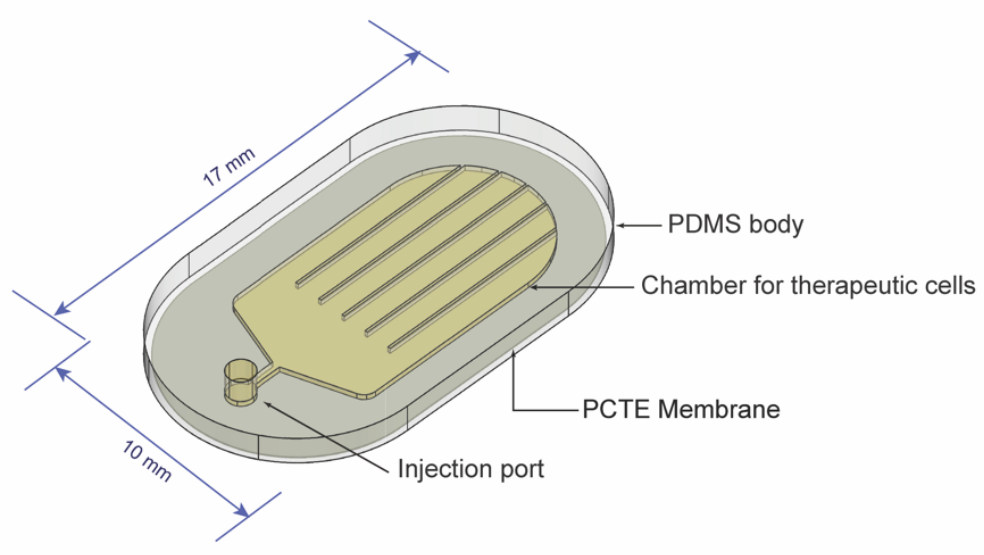

b Confocal microscopy imaging of the device

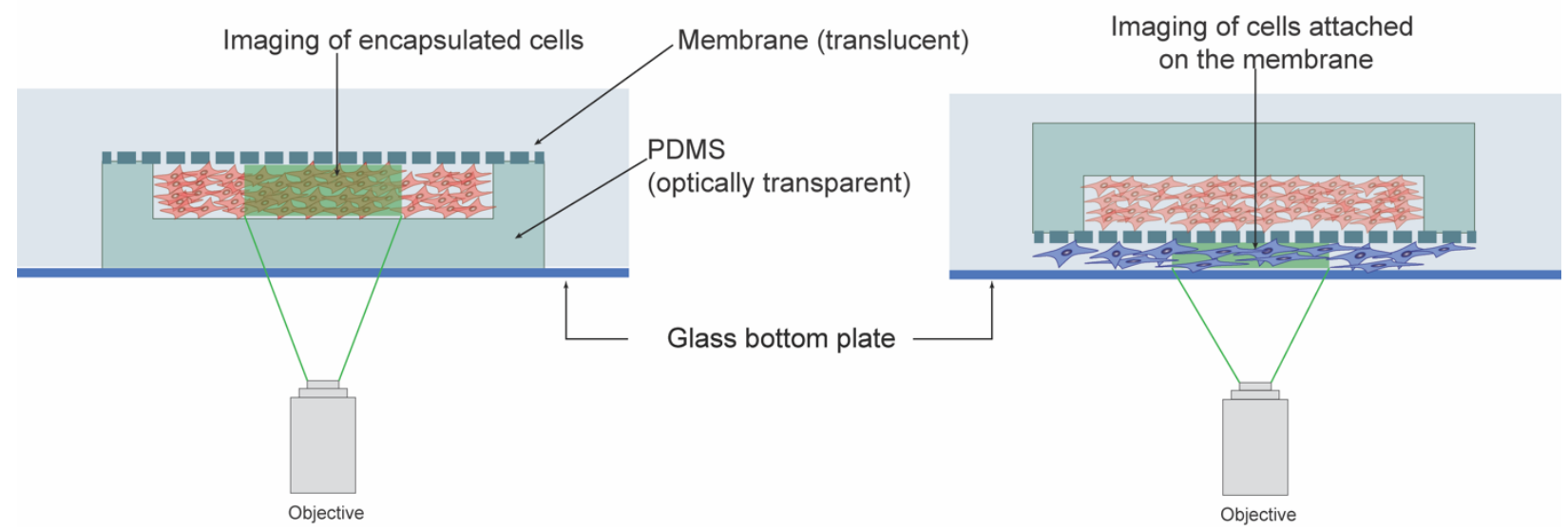

Figure S1: Design of the macrodevice. a) 3D CAD model of the device showing its various components. The PDMS is $1 \mathrm{~mm}$ thick while the cell chamber is 150 microns in height. The drawing is to scale and shown in isometric view. b) Schematic showing the experiential setup for imaging the encapsulated cells and the cells attached to the device. The PDMS being transparent allows for direct imaging of the cells inside the device, while the cells attached to the membrane can be imaged by placing the device membrane-side-down on the imaging platform. 


\section{Secretion rate of HEK-Epo cells}

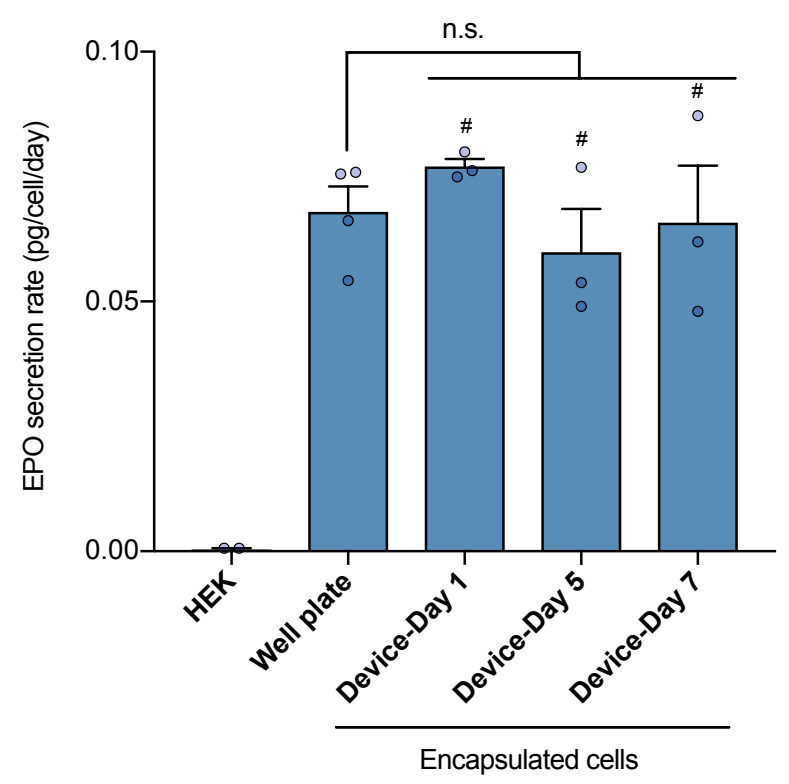

Figure S2: Secretion rate of EPO from native HEK cells and transformed cells (HEKEpo cells). Cells were cultured either free or within macrodevices in vitro, and the total amount of EPO in the culture supernatant over 24 hours was measured using ELISA assay. The total number was cells was measured using CellTiter Glo assay and used for normalizing EPO secretion values. Error bar: mean \pm s.e.m $(\mathrm{n}=3$ biological replicates). One-way ANOVA with Bonferroni multiple comparison correction. \# , P >0.999 indicated significance value for comparison with 'Well plate' group. 
a

Free Cells

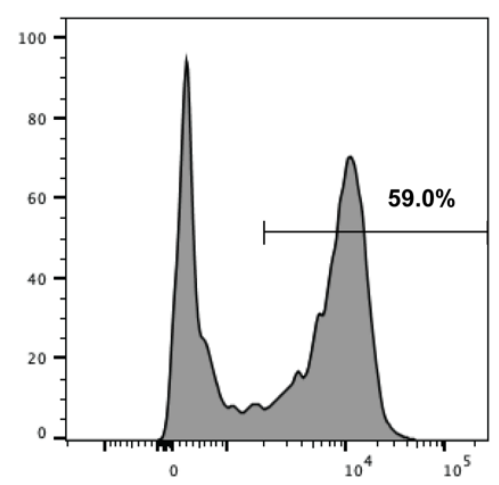

Day 1 after encapsulation

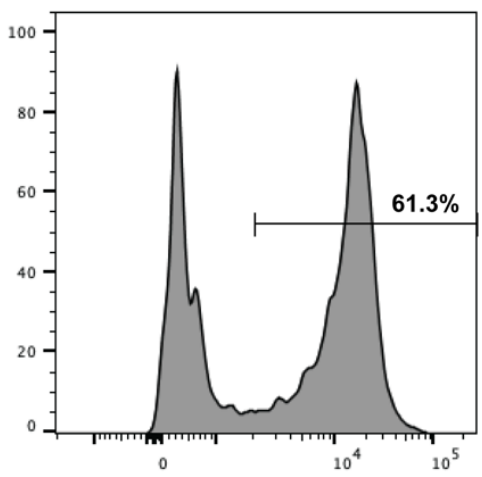

Day 7 after encapsulation

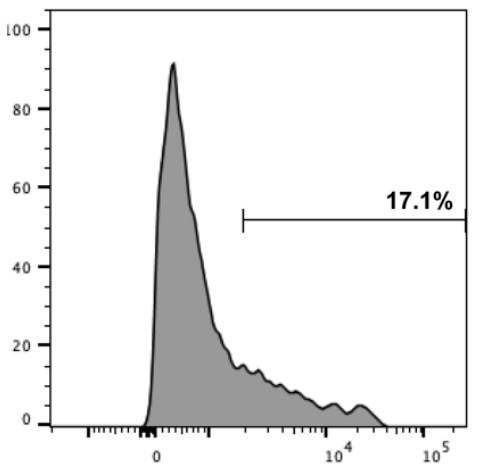

EdU-AF647

b

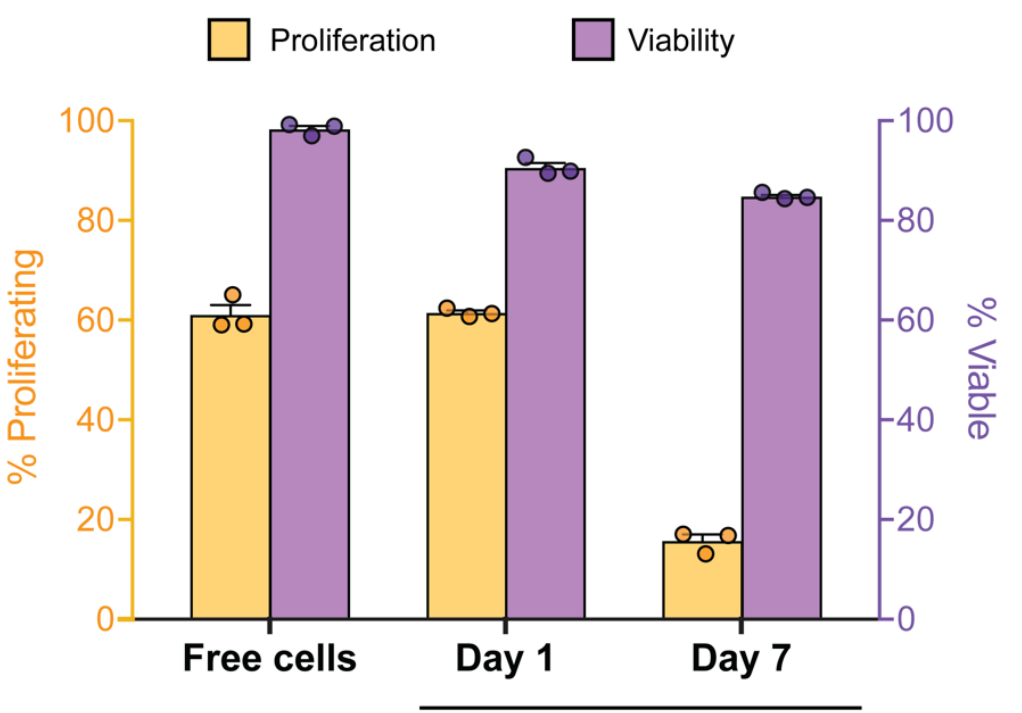

Post encapsulation

Figure S3: Proliferation and viability of the HEKepo cells inside the device. HEKepo cells were encapsulated inside macrodevices and cultured in vitro. Proliferation of cells was measured by assaying for the amount of EdU incorporated over $2 \mathrm{hr}(\mathrm{a})$, while viability was assayed using Sytox blue staining of the cells. a) Flow cytometry images of cells showing EdU incorporation within proliferating cells insude the macrodevice. Normal cells cultured in well plates (not encapsulated) is shown for comparison. b) Combined data of proliferation and viability of free and encapsulated cells. Error bars: mean \pm s.e.m $(n=3)$. 
Imaging cell escape from macrodevice
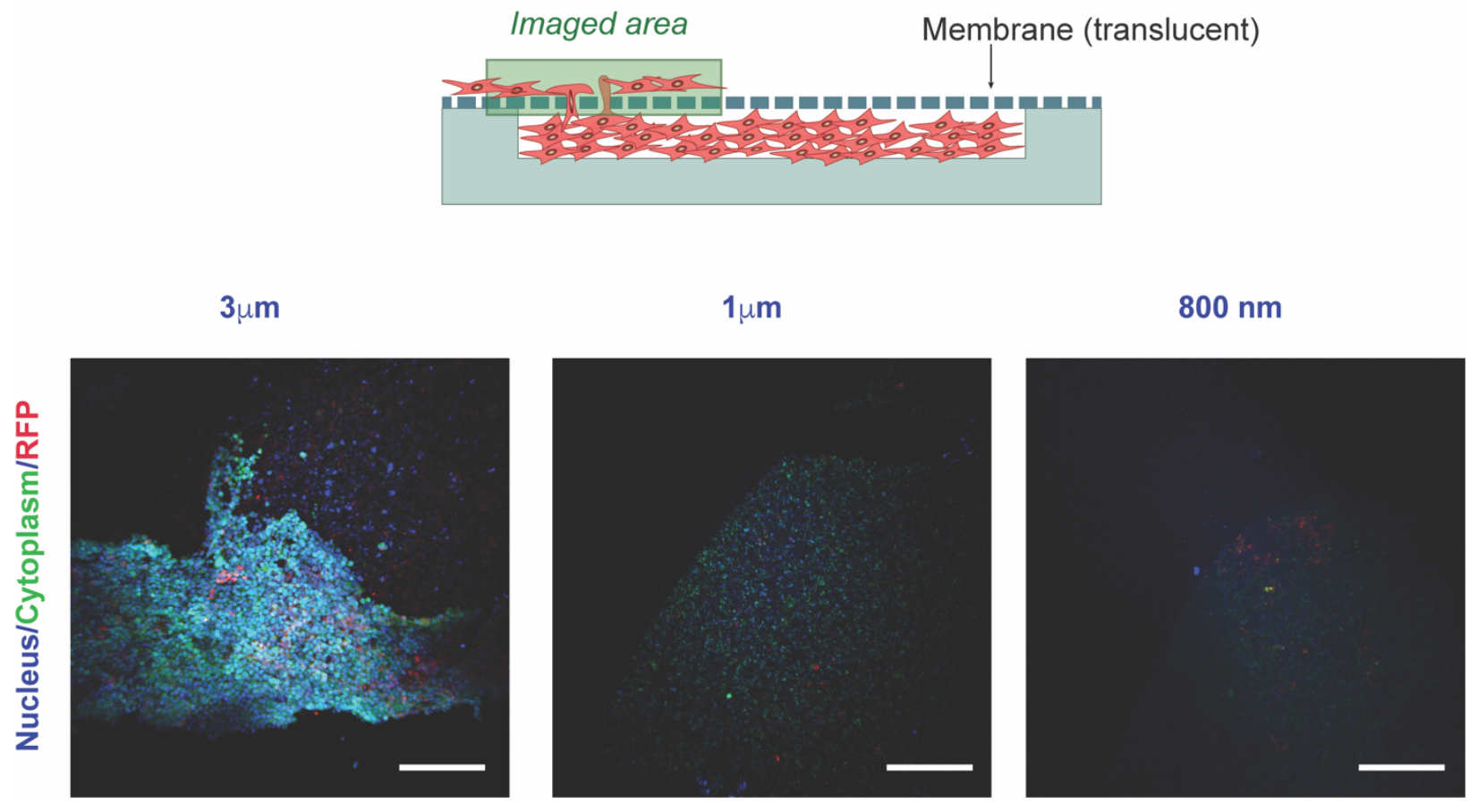

Figure S4: Escape of encapsulated cells from macrodevices. Macrodevices made with membranes of varying pore size $(0.8 \mu \mathrm{m}, 1 \mu \mathrm{m}$, and $3 \mu \mathrm{m})$ were loaded with $2 \times 10^{6} \mathrm{HEKepo}$ cells per device and cultured in vitro for one week. The cells were then stained with Calcein AM (cytosol/viability) and Hoechst 33342 (nucleus). Confocal microscopy was used to image the membrane surface as (shown in the schematic above) to identify cells escaping from of the membrane pores. Representative z-stacked images shown in the bottom pane relevel encapsulate cells escaping from the $3 \mu \mathrm{m}$ membranes (seen as the bright patch of cells growing on the membrane surface) while no cell escape was seen with $1 \mu \mathrm{m}$ or lower membranes. Experiments were repeated twice with similar results. Scale bar: $100 \mu \mathrm{m}$. 


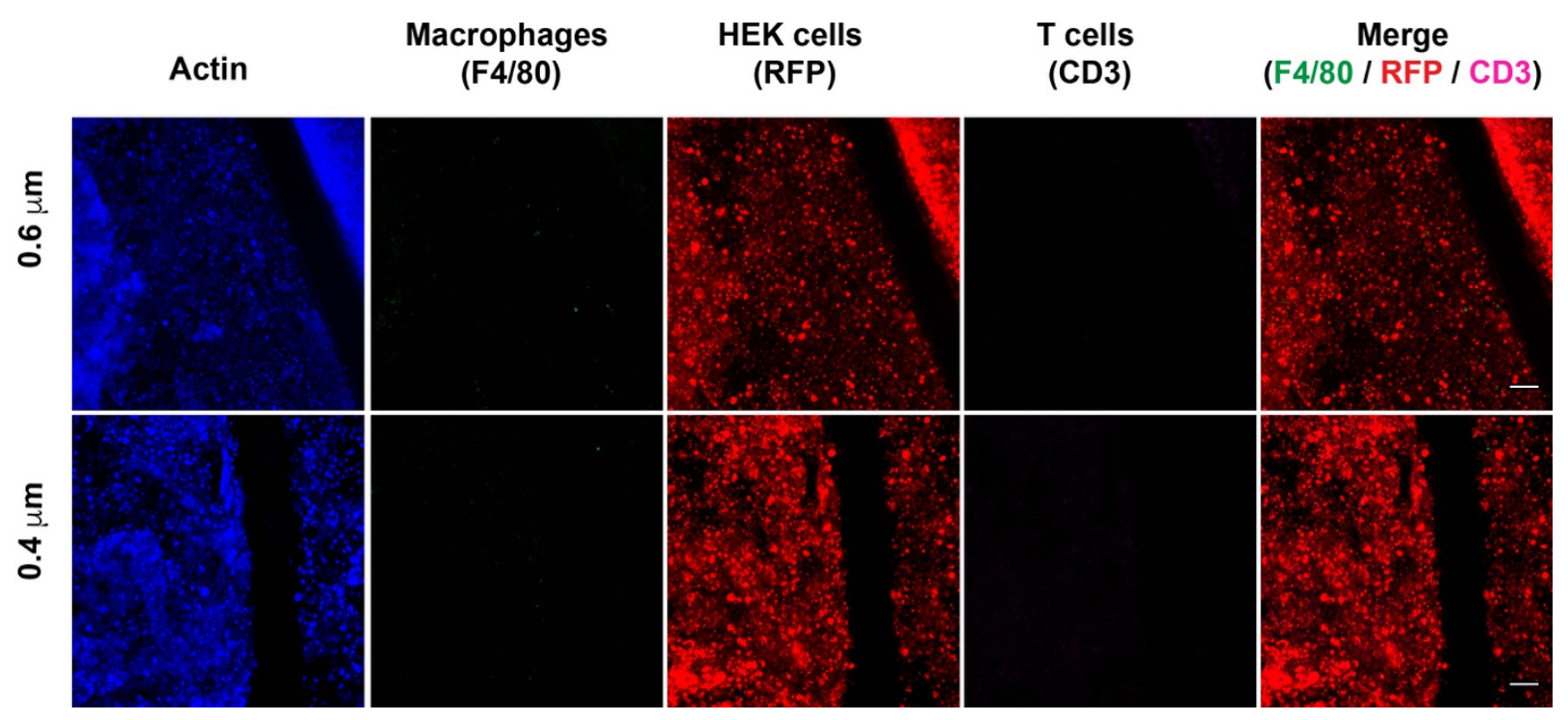

Figure S5: Representative z-stack images of devices in Figure 2 with membrane pore sizes of $0.6 \mu \mathrm{m}$ and $0.4 \mu \mathrm{m}$. Experiments were repeated twice with similar results. Scale bar $100 \mu \mathrm{m}$. 


\section{a}

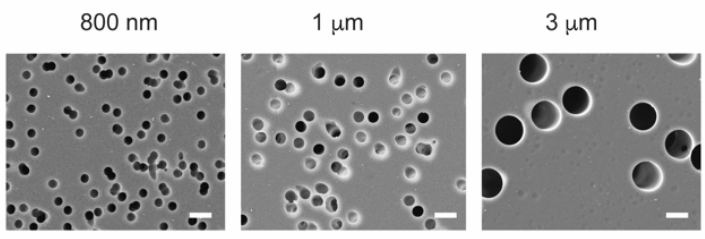

b

Pore-size distribution from SEM images

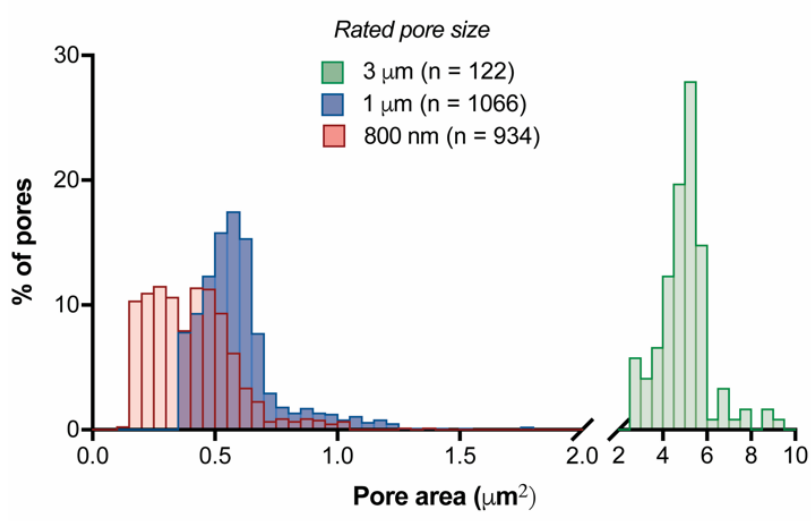

C

\begin{tabular}{cccc}
\hline \multirow{2}{*}{$\begin{array}{c}\text { Rated pore size } \\
(\text { Nominal) }[\mu \mathrm{m}]\end{array}$} & $\begin{array}{c}\text { Pore diameter }[\mu \mathrm{m}] \\
\text { (by bubble point) }\end{array}$ & \multicolumn{2}{c}{$\begin{array}{c}\text { Pore cross sectional area } \\
\text { measured using SEM imaging }\end{array}$} \\
\cline { 3 - 4 } & & Mean $\left[\mu \mathrm{m}^{2}\right]$ & $\operatorname{Max}_{\left[\mu \mathrm{m}^{2}\right]}$ \\
\hline 0.8 & $0.6-0.8$ & 0.4 & 1.05 \\
1.0 & $0.8-1.0$ & 0.55 & 1.25 \\
3.0 & $2.2-3.0$ & 5.0 & 9.0 \\
\hline *provided by manufacturer & \multicolumn{3}{c}{}
\end{tabular}

Figure S6: Estimation of pore-size distribution on $0.8 \mu \mathrm{m}, 1 \mu \mathrm{m}$, and $3 \mu \mathrm{m}$ membranes. a) SEM images of the membranes used for analysis. Scale bar $2 \mu \mathrm{m}$ b) Distribution of pore crosssectional area from SEM imaging. Measurements represents aggregate of three separate samples for each pore size, each sample imaged at five random locations. The total number of pores analyzed is indicated in the legend. c) A summary chart for the membrane measurements. Experiments were repeated twice with similar results 


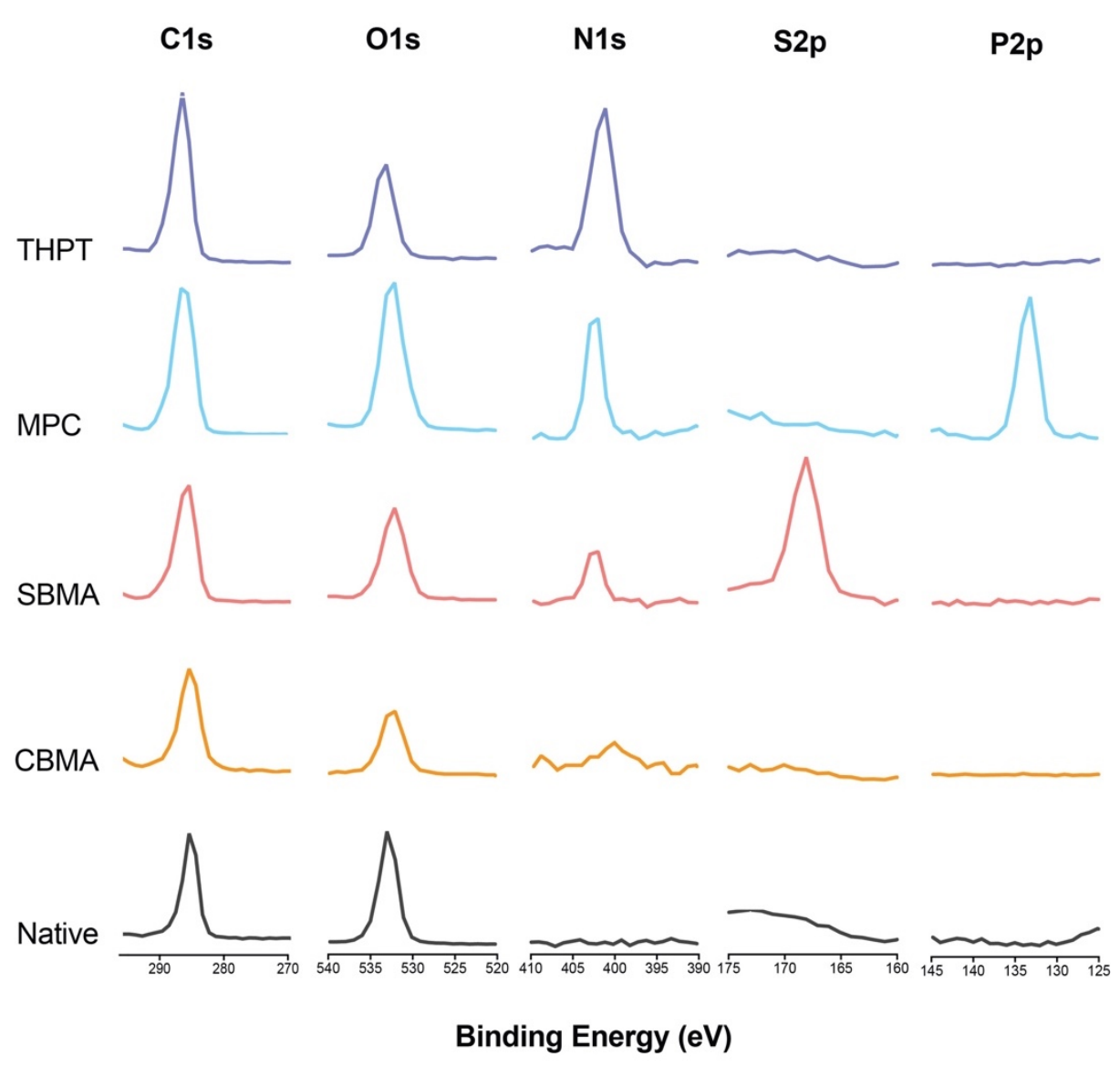

Figure S7: XPS spectrum of key elements (Carbon C, Oxygen O, Nitrogen N, Sulfur S, Phosphorus $\mathrm{P}$ ) obtained from analysis of device surface after ATRP modification. 


\section{Contact angle measurements}
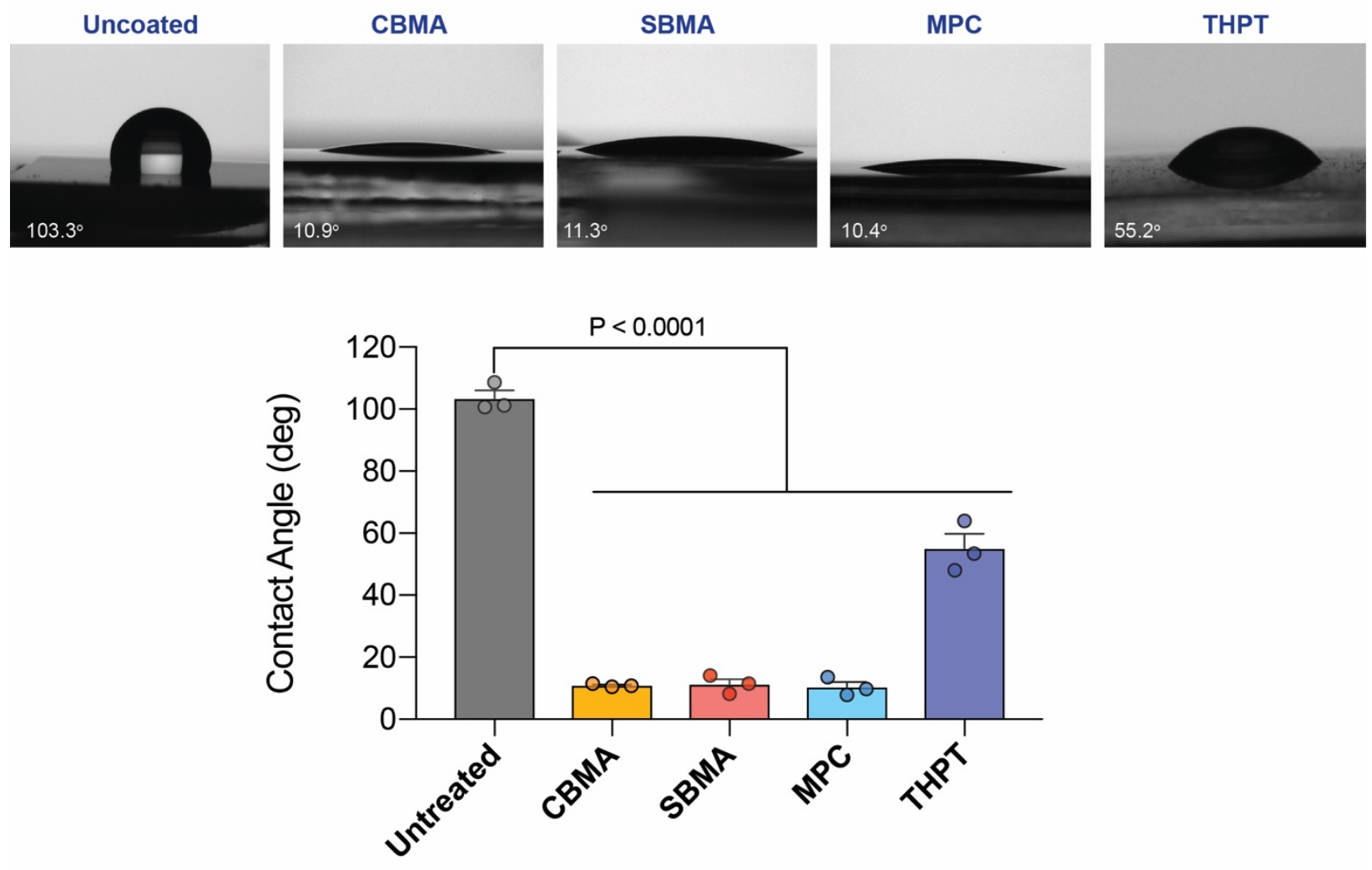

Figure S8: Effect of surface coating on hydrophilicity of the device. Contact angle was measured using the sessile drop technique on a DSA100 (Kruss). The top panel shows representative images of the sessile drop on the device surface. The bottom graph shows the summary of the measurements indicating significant reduction in contact angle, especially for the zwitterionic coatings. Error bar: mean \pm s.e.m $(n=3$ samples $)$. P-value was analyzed using one-way ANOVA with Bonferroni multiple comparison correction. 


\section{Scanning Electron Microcopy of surfaces after ATRP coating}
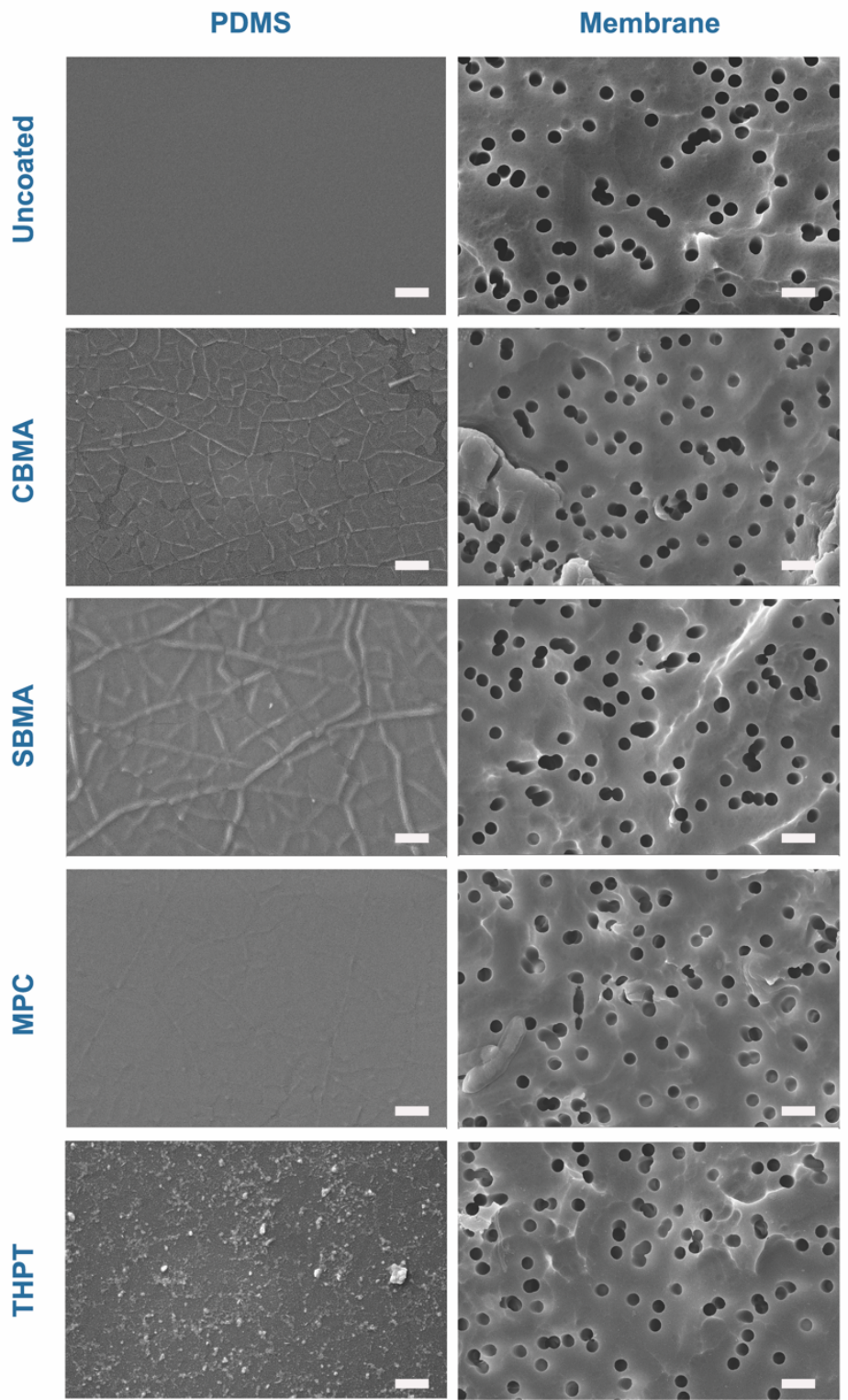

Figure S9: Surface analysis of the device using scanning electron microscopy (SEM). SEM images showing the surface of the macrodevices before and after the surface coating. The polymer coating on the surface can be clearly seen. The wrinkles on the zwitterionic coated devices (CBMA, SBMA, and MPC) is an artifact due to dehydration during the sample preparation process. Experiments were repeated thrice with similar results. Scale bar: $1 \mu \mathrm{m}$. 
a

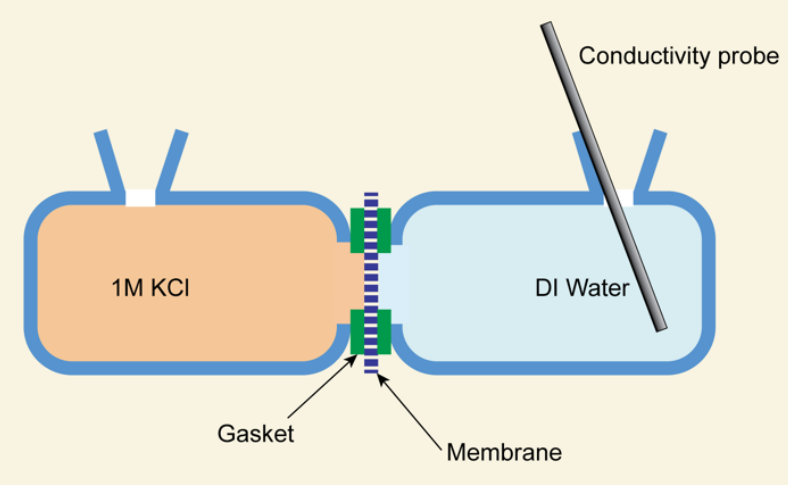

b

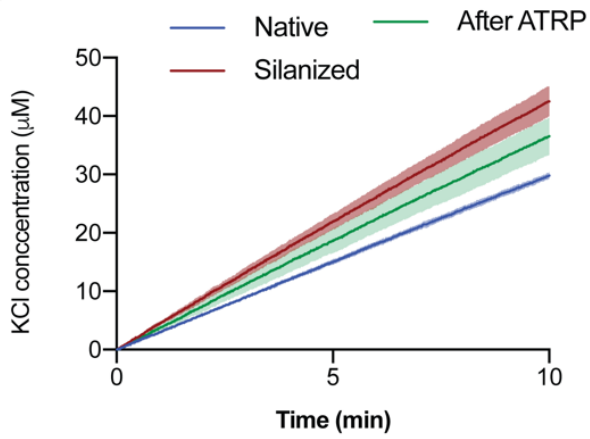

c

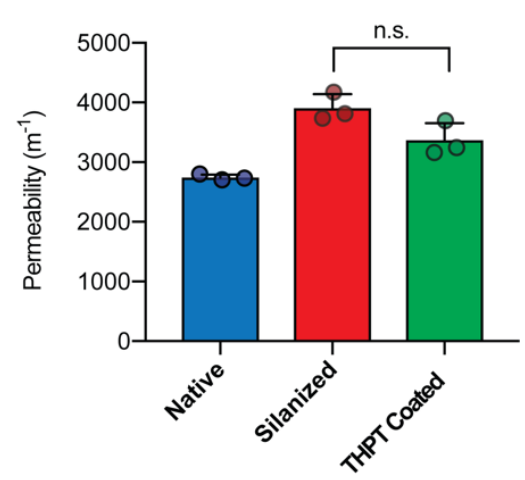

Figure S10: Measuring permeability of membranes. a) A horizontal Franz-diffusion cell setup for measuring membrane permeability. b) $\mathrm{KCl}$ concentration curves over time on the right side of the cell measured using the conductivity probe. c) Comparison of absolute membrane permeability of native (obtained from vendor), silanized (after APTES treatment as present on uncoated devices) and after THPT-coating. Error bar: mean \pm s.e.m $(n=3)$. Comparison done by two-tailed unpaired t-test; ns, $\mathrm{P}=0.0647$. 


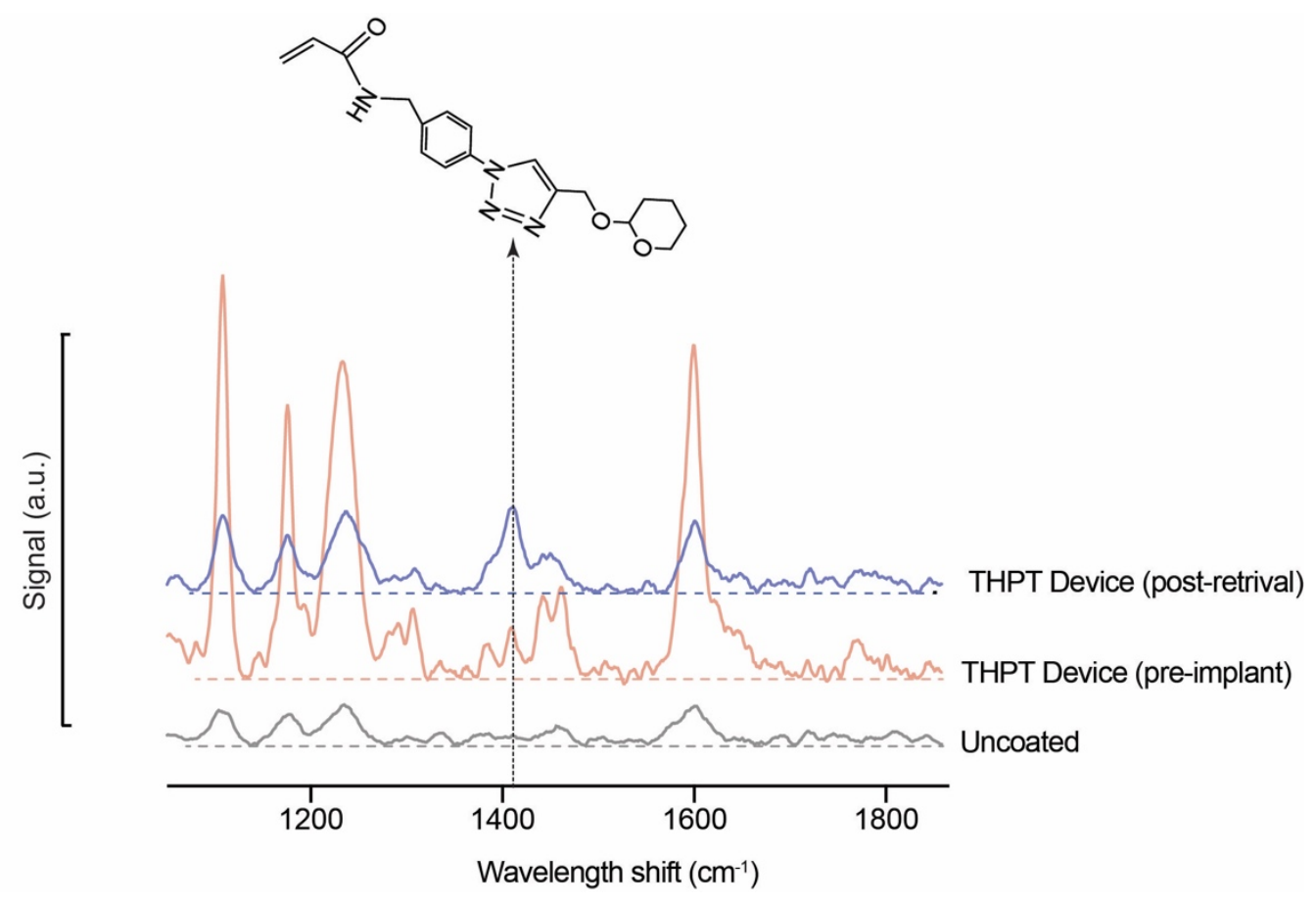

Figure S11: Confocal raman mapping of THPT-coated device membranes, retrieved 28 days after transplantation in i.p. of BALB/c mice. The peak $1410 \mathrm{~cm}^{-1}$ corresponding to the triazole ring can be seen on both the pre-transplant and explanted devices confirming that the THPT coating did not leach in vivo. Experiments were repeated twice with similar results. 
a

Serum antibody against HEK-cells in C57BL/6 mice

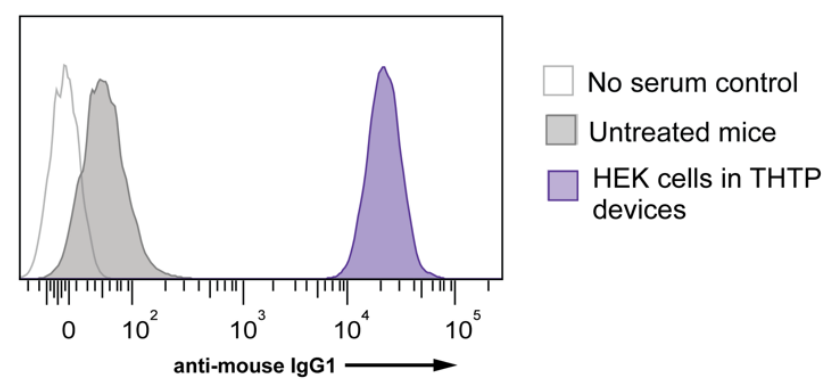

b

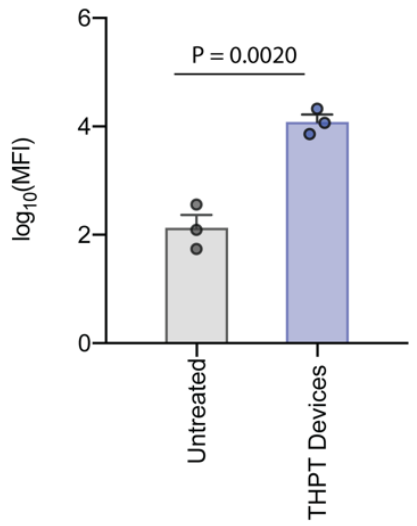

Figure S12: Detection of graft specific antibody in serum of C57BL/6 mice harboring THPTdevices with HEKepo cells. HEK cells incubated with animal serum (day 35) and counterstained with anti-mouse IgG-AF647 was analyzed using flow-cytometry (a) which showed the presence of cell specific antibodies in all the animals receiving the devices. (b) Quantification of mean fluorescence intensity (MFI) showed significant levels of anti-graft IgG with the devices over untreated animals. Error bars mean \pm s.e.m. $\mathrm{n}=3$ per group. Statistical analysis: two-tailed unpaired t-test.

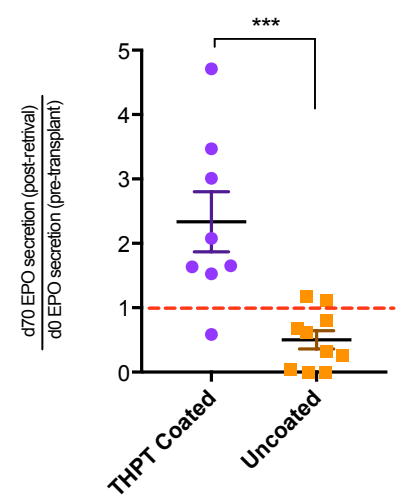

Figure S13: Fold change in EPO secretion rate of THPT-coated and uncoated devices prior to transplantation and after retrieval on day 70 post-transplantation. Experiment is detailed in Figure $5 e$, and EPO secretion was measured in vitro. Error bars: mean \pm s.e.m; $n=8$ (THPT coated devices) and 10 (uncoated devices). Statistical test: two-tailed unpaired t-test. ${ }^{* *}, \mathrm{P}=0.0008$. 
In-vitro charecterization of HEKepo-tetR cells

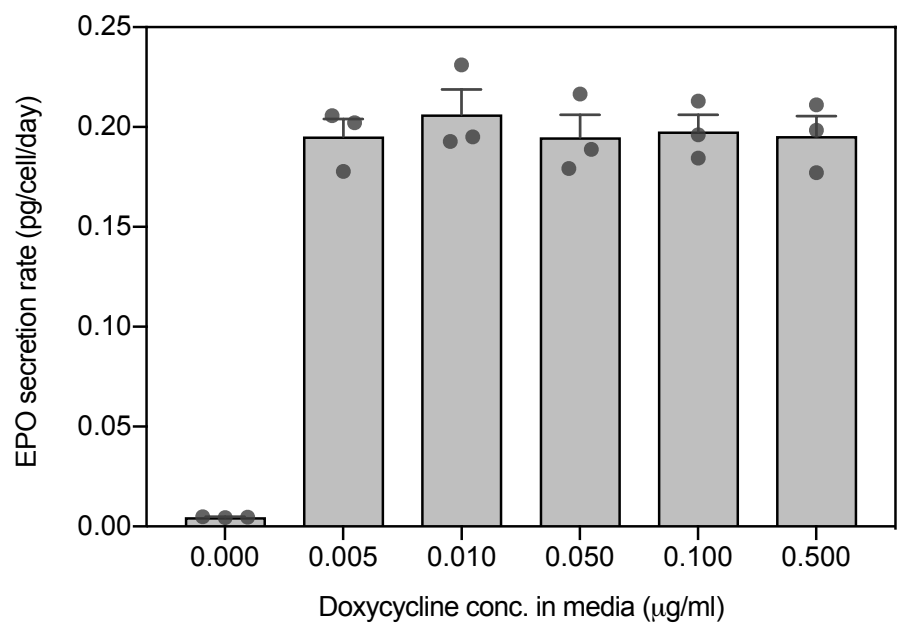

Figure S14: Secretion rate of EPO at different doxycycline concentration in media (treated for $48 \mathrm{hrs}$ ) from dox-inducible HEKepo cells used in Figure 5f. Error bars: mean \pm s.e.m ( $\mathrm{n}=3$ biological replicate).

Hematocrit

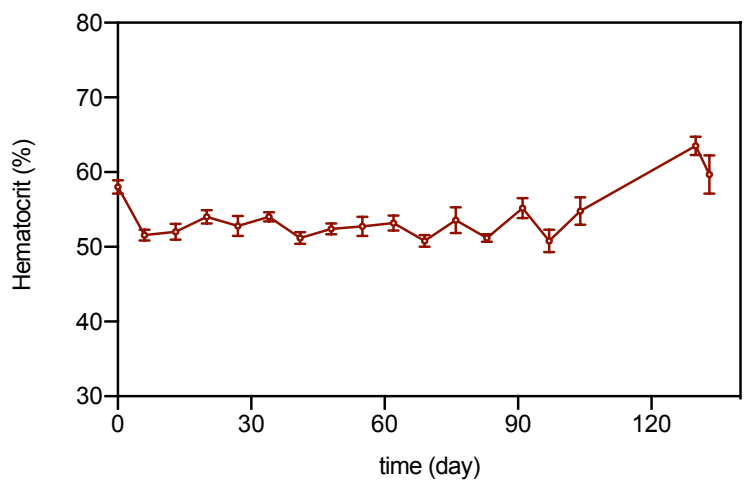

Figure S15: Hematocrit of animals receiving THPT-coated devices encapsulating doxycycline inducible HEKepo cells, sowing excellent control of hematocrit levels. Error bars: mean \pm s.e.m. $\mathrm{n}=5$ animals. 


\section{- THPT coated — Uncoated}

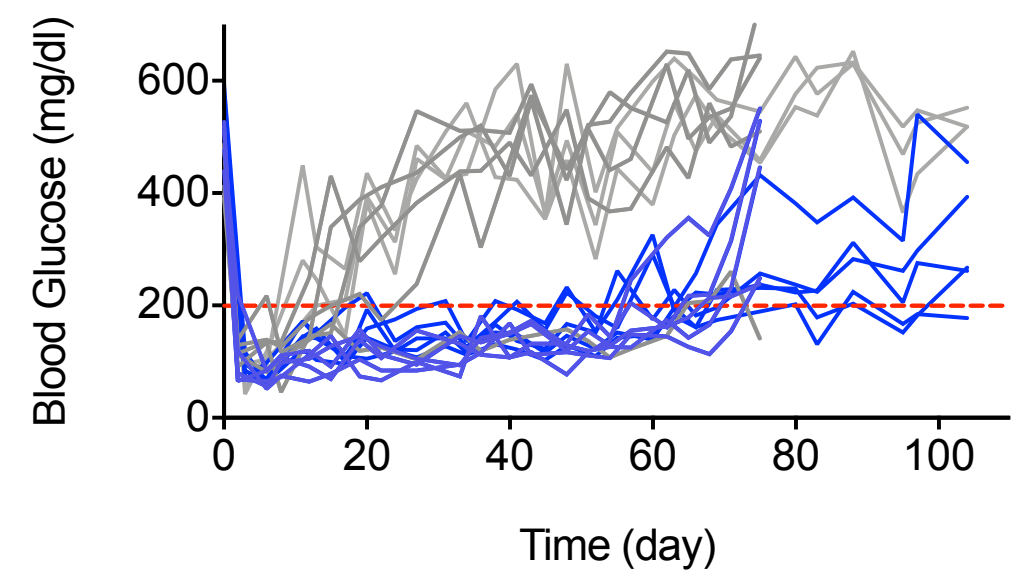

Figure S16: Individual BG plots of Figure 6. 
a

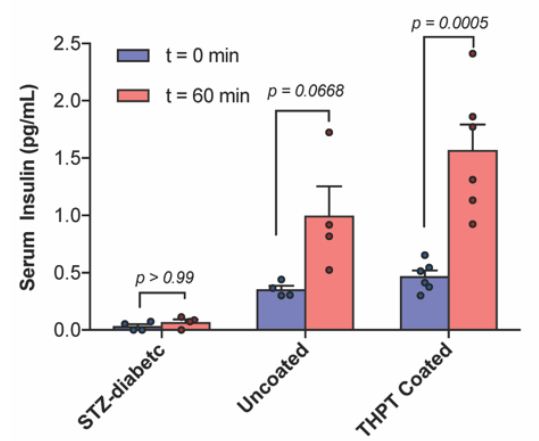

b

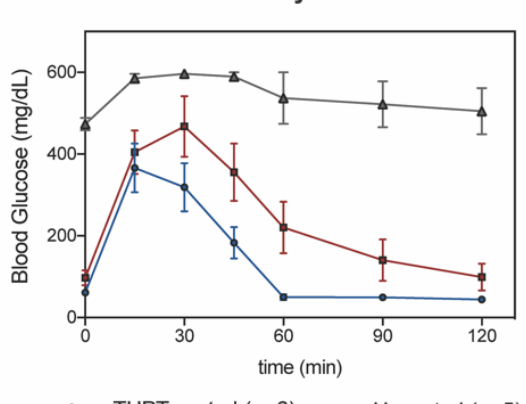

Day 7

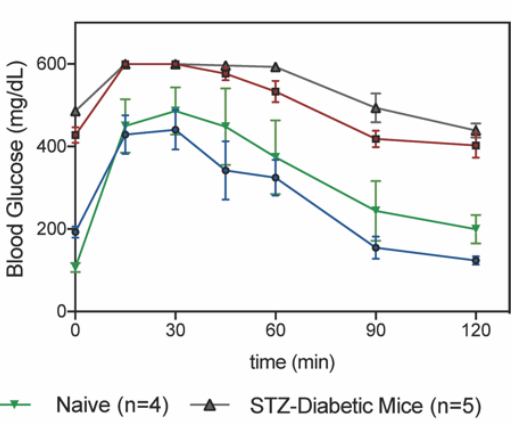

Figure S17: Additional Intravenous Glucose Tolerance Test (IVGTT) with transplanted rat islets inside macrodevices into STZ-diabetic C57BL/6 mice: a) Serum insulin levels during IVGTT on day 7 post transplantation shows significant rise in serum insulin levels in response to glucose in the treated mice groups. Error bar: mean \pm s.e.m. Group size $n=6$ animals (THPT), 4 animals (Uncoated and STZ-diabetic). Two-way ANOVA with Bonferroni multiple comparison correction. P-values are indicated on the graph. b) The blood glucose kinetic after glucose injection is shown for 7 and 21 days after transplantation. Error bar: mean \pm s.e.m. 


\section{Islets retrivals}

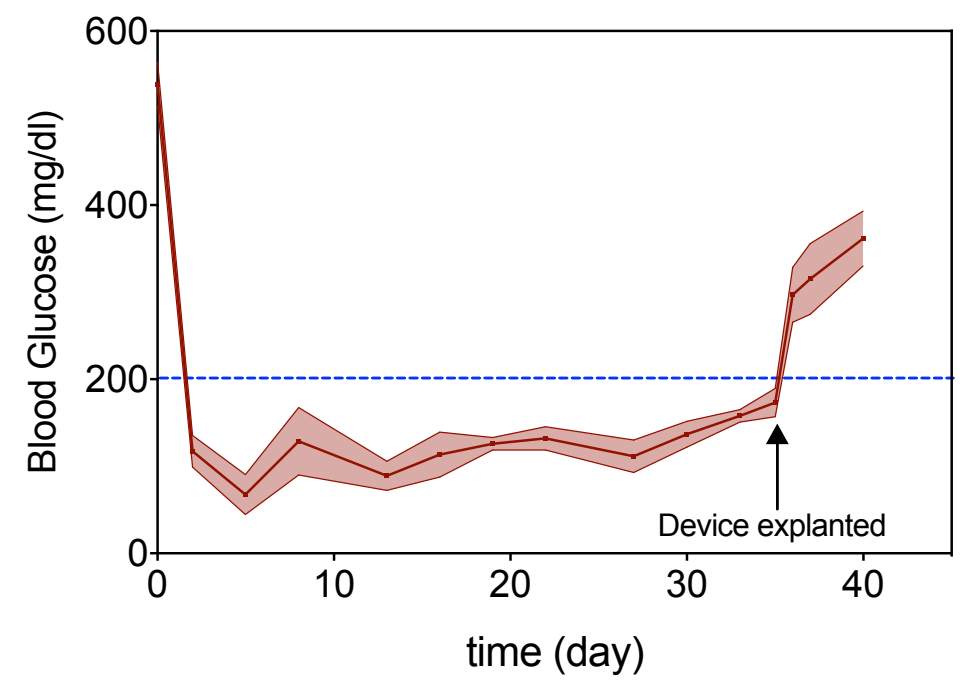

Figure S18: BG correction of STZ-diabetic C57BL/6 mice implanted with THPT-coated devices encapsulating 200 IEs rat islets, showing animals maintained euglycemia until devices were explanted (day 35, indicated by an arrow), after which the animals promptly became diabetic. Data points represents group mean $(n=5)$ while the shaded region is s.e.m. 


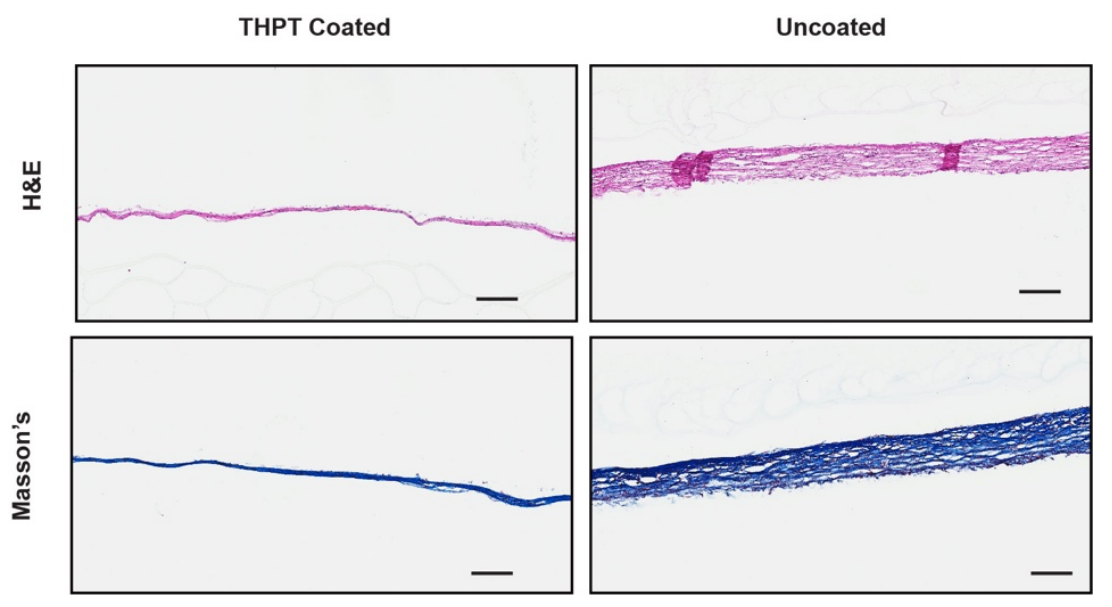

Figure S19: Representative histology images of the devices carrying rat islets retrieved from STZdiabetic C57BL/6 mice after 110d, showing the THPT-coated devices remained biocompatible with only a thin fibrotic capsule. Histological sections shows fibrosis on the device membrane with H\&E stain and masson's trichrome. Experiments were repeated twice with similar results. Scale bar: $100 \mu \mathrm{m}$. 


\section{Estimating membrane pore-size distribution}

Our studies with membranes having different pore-sizes demonstrated that controlling pore-size can change the intensity and type of immune cell infiltration inside the device. Specifically, we found that while membranes with $3 \mu \mathrm{m}$ pores allowed infiltration by T-cells and macrophages, pores with a rated diameter of $1 \mu \mathrm{m}$ allowed selective infiltration of macrophages, and $800 \mathrm{~nm}$ pores completely prevented cell infiltration. The results are significant since cell migration though pores below $7 \mu \mathrm{m}^{2}$ area ( $3 \mu \mathrm{m}$ diameter) has not been observed before. However, we wanted to accurately determine the pore-size distribution on membranes to ascertain the cut-off range for cell infiltration.

The polycarbonate tract-etched (PCTE) membranes used in this study contains straight pores of narrow diameter range. The pores are made by exposing thin sheets of PC to charged ions, creating defects or 'tracks' within the material, which are then chemically etched to obtain straight cylindrical pores. It is difficult to accurately characterize the pore structure and distribution of membranes, and the available methodologies have their own limitations. Bubble point measurement gives an accurate estimate of pore-diameters and is used by manufacturers to rate the PCTE membranes. The rated pore-size is the largest pore diameter present on the membrane. But this method underestimates the pore cross-section area since it does not accurately account for overlapping/merged pores present on membranes.

Scanning Electron Microscopy (SEM) imaging provides another method for estimating pore crosssection area (and size distribution) which might be more relevant for cell migration. Therefore, we used this method to further characterize the membranes used in this study. Membranes were coated with $2 \mathrm{~nm}$ of gold using a sputter coater and imaged on a Zeiss Crossbeam 540 microscope at $5 \mathrm{kV}$ beam. Three separate membranes for each pore size was images at five random locations. The images were analyzed using ImageJ software, where they were filtered using an intensity threshold to identify the pores, then the pore area was calculated using the inbuilt Analyze Particle function.

The results of our analysis are shown in Figure S6. We found that while majority of pores were within the rated size (based on the diameter) about $10-15 \%$ of pores with larger sizes were present on all membranes. Pores up to $1 \mu \mathrm{m}^{2}$ in area were found on the $800 \mathrm{~nm}$ membranes indicating that this might be the size-cutoff for preventing cell infiltration. Although a majority of pores on the $1 \mu \mathrm{m}$ membranes overlapped in size with those on the $800 \mathrm{~nm}$ membranes, about $4 \%$ of pores were of larger area. Hence it seems likely that these pores in the size range of $1-1.25 \mu \mathrm{m}^{2}$ area were responsible for the selective infiltration of macrophages as seen with the $1 \mu \mathrm{m}$ membranes. 